\title{
Level densities of heavy nuclei in the shell model Monte Carlo approach
}

\author{
Y. Alhassid ${ }^{1, a}$, G.F. Bertsch ${ }^{2,3}$, C.N. Gilbreth ${ }^{3}$, H. Nakada ${ }^{4}$, and C. Özen ${ }^{5}$ \\ ${ }^{1}$ Center for Theoretical Physics, Sloane Physics Laboratory, Yale University, New Haven, Connecticut 06520 , \\ USA \\ ${ }^{2}$ Department of Physics, University of Washington, Seattle, Washington 98915, USA \\ ${ }^{3}$ Institute for Nuclear Theory, Box 351560, University of Washington, Seattle, Washington 98915, USA \\ ${ }^{4}$ Department of Physics, Graduate School of Science, Chiba University, Inage, Chiba 263-8522, Japan \\ ${ }^{5}$ Faculty of Engineering and Natural Sciences, Kadir Has University, Istanbul 34083, Turkey
}

\begin{abstract}
Nuclear level densities are necessary input to the Hauser-Feshbach theory of compound nuclear reactions. However, the microscopic calculation of level densities in the presence of correlations is a challenging many-body problem. The configurationinteraction shell model provides a suitable framework for the inclusion of correlations and shell effects, but the large dimensionality of the many-particle model space has limited its application in heavy nuclei. The shell model Monte Carlo method enables calculations in spaces that are many orders of magnitude larger than spaces that can be treated by conventional diagonalization methods and has proven to be a powerful tool in the microscopic calculation of level densities. We discuss recent applications of the method in heavy nuclei.
\end{abstract}

\section{Introduction}

Level densities play an important role in the Hauser-Feshbach theory [1] of compound nuclear reactions, but are not always accessible by direct measurements. The calculation of level densities in the presence of correlations is a challenging many-body problem. Most approaches are based on empirical modifications of the Fermi gas formula or on mean-field approximations [2] that can often miss important correlations. The configuration-interaction (CI) shell model approach accounts for correlations, but conventional diagonalization methods are limited to spaces of dimensionality $\sim 10^{11}$. The dimension of the many-particle model space increases combinatorially with the numbers of valence nucleons and single-particle orbitals, hindering applications of the CI shell model approach to mid-mass and heavy nuclei.

The auxiliary-field quantum Monte Carlo method, also known in nuclear physics as the shell model Monte Carlo (SMMC) method [3-6], enables microscopic calculations in spaces that are many orders of magnitude larger (e.g., $\sim 10^{30}$ in recent applications to rare-earth nuclei) than those that can be treated by conventional methods.

ae-mail: yoram.alhassid@yale.edu 
The outline is as follows. In Sec. 2 we briefly review the SMMC method. In Sec. 3 we discuss the emergence of collectivity in heavy nuclei within the framework of the CI shell model. In Sec. 4 we present results for the state densities in families of lanthanide isotopes and find them to be good agreement with experimental data. In Sec. 5 we benchmark finite-temperature mean-field approximations to level densities by comparing them with SMMC state densities. In Sec. 6 we discuss nuclear deformation in the CI shell model. Deformation is an important concept for understanding heavy nuclei but it is usually introduced in a mean-field approximation that breaks rotational symmetry. Here we discuss how the intrinsic quadrupole deformation distributions can be derived in a framework that preserves rotational symmetry. We conclude in Sec. 7.

\section{Shell model Monte Carlo method}

\subsection{Hubbard-Stratonovich transformation}

The SMMC method is based on the Hubbard-Stratonovich (HS) transformation [7], in which the Gibbs ensemble $e^{-\beta \hat{H}}$ at inverse temperature $\beta=1 / T$ for a nucleus described by an Hamiltonian $\hat{H}$ can be viewed as an imaginary-time propagator and written as a functional integral over propagators of non-interacting particles in external time-dependent auxiliary fields $\sigma(\tau)$

$$
e^{-\beta \hat{H}}=\int \mathcal{D}[\sigma] G_{\sigma} \hat{U}_{\sigma} .
$$

Here $G_{\sigma}$ is a Gaussian factor and $\hat{U}_{\sigma}$ is a many-particle propagator for non-interacting particles for a given configuration of the auxiliary fields. The thermal expectation value of observable $\hat{O}$ is given by

$$
\langle\hat{O}\rangle=\frac{\operatorname{Tr}\left(\hat{O} e^{-\beta \hat{H}}\right)}{\operatorname{Tr}\left(e^{-\beta \hat{H}}\right)}=\frac{\int \mathcal{D}[\sigma] W_{\sigma}\langle\hat{O}\rangle_{\sigma} \Phi_{\sigma}}{\int \mathcal{D}[\sigma] W_{\sigma} \Phi_{\sigma}},
$$

where $W_{\sigma}=G_{\sigma}\left|\operatorname{Tr} \hat{U}_{\sigma}\right|$ is a positive-definite function, $\Phi_{\sigma}=\operatorname{Tr} \hat{U}_{\sigma} /\left|\operatorname{Tr} \hat{U}_{\sigma}\right|$ is the Monte Carlo sign function and $\langle\hat{O}\rangle_{\sigma} \equiv \operatorname{Tr}\left(\hat{O} \hat{U}_{\sigma}\right) / \operatorname{Tr} \hat{U}_{\sigma}$. The grand canonical traces in the integrands of Eq. (2) can be calculated in terms of the single-particle representation $\mathbf{U}_{\sigma}$ (an $N_{s} \times N_{s}$ matrix, where $N_{s}$ is the number of single-particle orbitals) of $\hat{U}_{\sigma}$. The trace of $\hat{U}_{\sigma}$ is given by

$$
\operatorname{Tr} \hat{U}_{\sigma}=\operatorname{det}\left(\mathbf{1}+\mathbf{U}_{\sigma}\right),
$$

while the grand canonical expectation value of a one-body operator $\hat{O}=\sum_{i, j} O_{i j} a_{i}^{\dagger} a_{j}$ is given by

$$
\left\langle a_{i}^{\dagger} a_{j}\right\rangle_{\sigma} \equiv \frac{\operatorname{Tr}\left(a_{i}^{\dagger} a_{j} \hat{U}_{\sigma}\right)}{\operatorname{Tr} \hat{U}_{\sigma}}=\left[\frac{1}{\mathbf{1}+\mathbf{U}_{\sigma}^{-1}}\right]_{j i} .
$$

The grand canonical expectation value of a two-body observable can be calculated using Wick's theorem.

\subsection{Canonical projection}

In the finite nucleus it is important to consider the canonical ensemble of fixed number of protons and neutrons. The particle-projected partition function for $\mathcal{A}$ particles is given by a discrete Fourier transform [8]

$$
\operatorname{Tr}_{\mathcal{A}} U_{\sigma}=\frac{e^{-\beta \mu \mathcal{A}}}{N_{s}} \sum_{m=1}^{N_{s}} e^{-i \varphi_{m} \mathcal{A}} \operatorname{det}\left(\mathbf{1}+e^{i \varphi_{m}} e^{\beta \mu} \mathbf{U}_{\sigma}\right)
$$


where $\varphi_{m}=2 \pi m / N_{s} \quad\left(m=1, \ldots, N_{s}\right)$ are quadrature points and $\mu$ is a chemical potential introduced to stabilize the numerical evaluation of the Fourier sum. Similarly for a one-body observable $\hat{O}=$ $\sum_{i, j} O_{i j} a_{i}^{\dagger} a_{j}$

$$
\operatorname{Tr}_{\mathcal{A}}\left(\hat{O} \hat{U}_{\sigma}\right)=\frac{e^{-\beta \mu \mathcal{A}}}{N_{s}} \sum_{m=1}^{N_{s}} e^{-i \varphi_{m} \mathcal{A}} \operatorname{det}\left(\mathbf{1}+e^{i \varphi_{m}+\beta \mu} \mathbf{U}_{\sigma}\right) \operatorname{tr}\left(\frac{1}{\mathbf{1}+e^{-i \varphi_{m}-\beta \mu} \mathbf{U}_{\sigma}^{-1}} \mathbf{O}\right)
$$

where $\mathbf{O}$ is the matrix with elements $O_{i j}$.

\subsection{Monte Carlo sampling}

In SMMC we choose a set of uncorrelated auxiliary-field configurations $\sigma^{(k)}$ from the positive-definite weight function $W_{\sigma}$. The expectation value of an observable in Eq. (2) is then estimated from

$$
\langle\hat{O}\rangle \approx \frac{\sum_{k}\langle\hat{O}\rangle_{\sigma^{(k)}} \Phi_{\sigma^{(k)}}}{\sum_{k} \Phi_{\sigma^{(k)}}}
$$

The statistical error of $\langle\hat{O}\rangle$ can be estimated from the variance of the "measurements" $\langle\hat{O}\rangle_{\sigma^{(k)}}$.

\section{Collectivity in heavy nuclei in the $\mathrm{Cl}$ shell model}

The single-particle model space we use for rare-earth nuclei is composed of the orbitals $0 g_{7 / 2}, 1 d_{5 / 2}, 1 d_{3 / 2}, 2 s_{1 / 2}, 0 h_{11 / 2}, 1 f_{7 / 2}$ for protons and $0 h_{11 / 2}, 0 h_{9 / 2}, 1 f_{7 / 2}, 1 f_{5 / 2}, 2 p_{3 / 2}, 2 p_{1 / 2}, 0 i_{13 / 2}, 1 g_{9 / 2}$ for neutrons. The single-particle levels are taken from a Woods-Saxon potential plus a spin-orbit interaction. The interaction includes attractive monopole pairing plus attractive multipole-multipole terms with quadrupole, octupole and hexadecupole components. The interaction parameters are determined empirically as discussed in Refs. [9] and [10].

Quantum Monte Carlo methods are often limited by the so-called sign problem, which leads to large statistical errors. However, the dominant components of effective nuclear shell model interactions [11] have a good sign (in the grand canonical ensemble) and are usually sufficient for realistic calculations of statistical and collective properties of nuclei. Small bad sign components of the interaction can be treated using the extrapolation method of Ref. [4]. The interaction discussed above has a good Monte Carlo sign in the grand canonical ensemble and the sign remains good in the canonical ensemble for even-even nuclei. For even-odd nuclei there is a moderate sign problem at high and intermediate temperatures that becomes more severe at low temperatures [12].

Heavy nuclei are known to exhibit various types of collectivity that are well described by empirical models. An important question is whether such collectivity, and, in particular, rotational collectivity, can be described in a truncated spherical shell model approach. Various types of collectivity are usually identified by their characteristic spectra of low-lying states. However, it is a challenge to obtain detailed spectroscopic information in SMMC. We found that the type of collectivity can be characterized by the low-temperature behavior of the thermal observable $\left\langle\mathbf{J}^{2}\right\rangle_{T}$, where $\mathbf{J}$ is the total angular momentum of the nucleus. For an even-even nucleus, we find (at low temperatures)

$$
\left\langle\mathbf{J}^{2}\right\rangle_{T} \approx\left\{\begin{array}{cc}
30 \frac{e^{-E_{2^{+}} / T}}{\left(1-e^{-E_{2} / T}\right)^{2}} & \text { vibrational band } \\
\frac{6}{E_{2^{+}}} T & \text { rotational band }
\end{array}\right.
$$

where $E_{2^{+}}$is the excitation energy of the lowest $2^{+}$level. 
In Fig. 1, we show the low-temperature behavior of $\left\langle\mathbf{J}^{2}\right\rangle_{T}$ vs. $T$ for ${ }^{162}$ Dy (left panel) and ${ }^{148} \mathrm{Sm}$ (right panel). The results are consistent with ${ }^{162} \mathrm{Dy}$ being a rotational nucleus, and ${ }^{148} \mathrm{Sm}$ a vibrational nucleus (see figure caption for details).
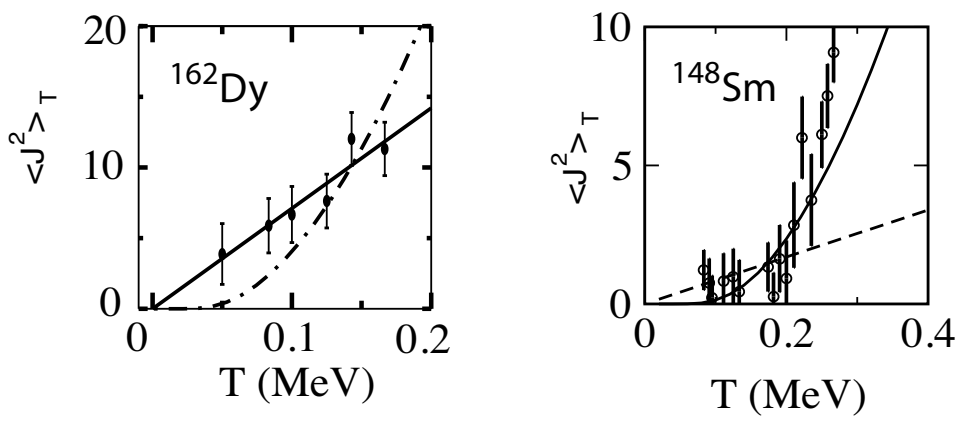

Figure 1. Left panel: $\left\langle\mathbf{J}^{2}\right\rangle_{T}$ vs. $T$ for ${ }^{162}$ Dy. The SMMC results (solid circles) fit well the rotational model (solid line) but not the vibrational model (dashed-dotted line). Adapted from Ref. [9]. Right panel: $\left\langle\mathbf{J}^{2}\right\rangle_{T}$ vs. $T$ for ${ }^{148} \mathrm{Sm}$ are in better agreement with the vibrational model (solid line) than the rotational model (dashed line). Taken from Ref. [13].

\subsection{Crossover from vibrational to rotational collectivity}

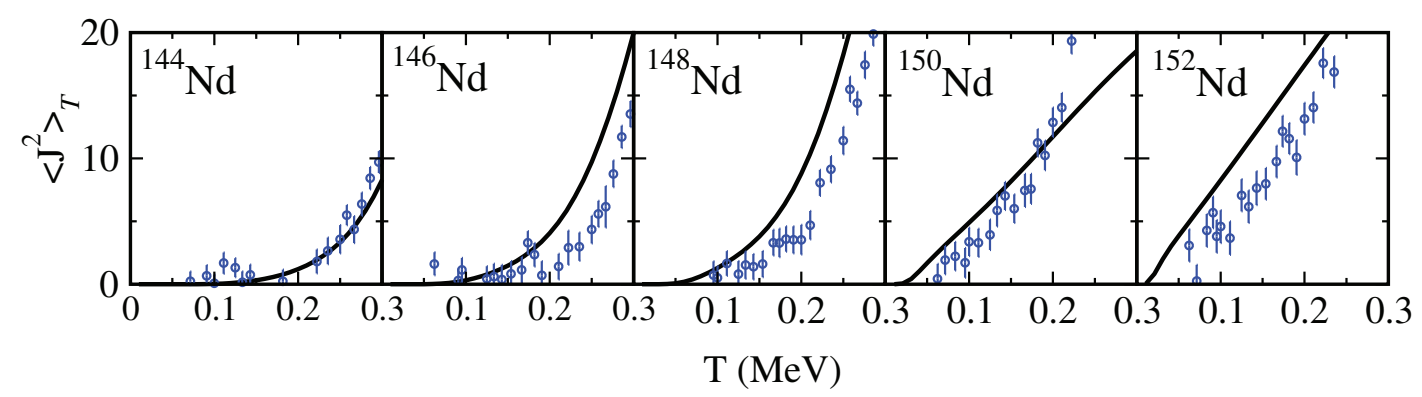

Figure 2. $\left\langle\mathbf{J}^{2}\right\rangle_{T}$ vs. $T$ for a family of even-even neodymium isotopes ${ }^{144-152} \mathrm{Nd}$. The SMMC results (open circles) are compared with results extracted from experimental data (solid lines, see text).

The observable $\left\langle\mathbf{J}^{2}\right\rangle_{T}$ can also be used to describe the crossover from vibrational to rotational collectivity a family of isotopes. Fig. 2 shows this observable vs. $T$ in a family of even-even neodymium isotopes. We observe a crossover from a soft response to temperature in the vibrational nucleus ${ }^{146} \mathrm{Nd}$ to a rigid behavior in the rotational nucleus ${ }^{152} \mathrm{Nd}$. The solid lines are determined from experimental data using

$$
\left\langle\mathbf{J}^{2}\right\rangle_{T}=\frac{1}{Z(T)}\left(\sum_{i}^{N} J_{i}\left(J_{i}+1\right)\left(2 J_{i}+1\right) e^{-E_{i} / T}+\int_{E_{N}}^{\infty} d E_{x} \rho\left(E_{x}\right)\left\langle\mathbf{J}^{2}\right\rangle_{E_{x}} e^{-E_{x} / T}\right),
$$


where the partition function $Z(T)$ is

$$
Z(T)=\sum_{i}^{N}\left(2 J_{i}+1\right) e^{-E_{i} / T}+\int_{E_{N}}^{\infty} d E_{x} \rho\left(E_{x}\right) e^{-E_{x} / T} .
$$

The summations in Eqs. (9) and (10) are over a complete set of experimentally measured levels with energies $E_{i}$ and spins $J_{i}$ up to an energy of $E_{N}$. Above $E_{N}$ we use a back-shifted Fermi gas level density $\rho\left(E_{x}\right)$ with parameters determined from level counting at low excitation energies and neutron resonance data at the neutron separation energy.

\section{State densities in lanthanide isotopes}

SMMC has been a powerful method for calculating state densities in the presence of correlations $[9,10,14-18]$. In SMMC we calculate the canonical energy vs. inverse temperature $\beta$ as an observable $E_{c}(\beta)=\langle\hat{H}\rangle$. The canonical partition function $Z_{c}(\beta)$ is then determined by integrating the thermodynamic relation $E_{c}(\beta)=-\partial \ln Z_{c} / \partial \beta$. The level density is related to the partition function by an inverse Laplace transform

$$
\rho(E)=\frac{1}{2 \pi i} \int_{-i \infty}^{i \infty} d \beta e^{\beta E} Z_{c}(\beta) .
$$

The average state density is obtained by calculating Eq. (11) in the saddle-point approximation [19]

$$
\rho(E) \approx\left(2 \pi\left|\frac{\partial E}{\partial \beta}\right|\right)^{-1 / 2} e^{S_{c}(\beta)}
$$

where $S_{c}(E)=\ln Z_{c}+\beta E_{c}$ is the canonical entropy. The inverse temperature $\beta$ is determined as a function of $E$ from the saddle-point condition $E_{c}(\beta)=E$.

In Fig. 3 we show the SMMC state densities (open circles) in families of even-mass samarium and neodymium isotopes. They are in good agreement with level counting data at low excitation energies (histograms) and neutron resonance data (triangles) [21] when available.

\section{Mean-field approximations to level densities}

In SMMC we take into account all correlations within the CI shell model space. However, these calculations are computationally intensive and it is interesting to determine the accuracy of meanfield approximations to level densities. Recently we used SMMC level densities to benchmark finitetemperature mean-field approximations to level densities in a strongly deformed nucleus ${ }^{162} \mathrm{Dy}$ and in a spherical nucleus ${ }^{148} \mathrm{Sm}$ with a strong pairing condensate [22].

A finite-temperature mean-field theory such as the Hartree-Fock (HF) and the Hartree-FockBogoliubov (HFB) approximations work in the grand canonical ensemble while the SMMC calculations are canonical with fixed numbers of protons and neutrons. It is therefore necessary to carry out an approximate particle-number projection in the mean-field theory and this is usually done in the saddle-point approximation. The saddle-point approximation leads to a three-dimensional Jacobian $\left|\partial\left(E, N_{p}, N_{n}\right) / \partial\left(\beta, \alpha_{p}, \alpha_{n}\right)\right|$ (where $\alpha_{p} / \beta$ and $\alpha_{n} / \beta$ are chemical potentials for $N_{p}$ protons and $N_{n}$ neutrons) as a pre-exponential factor to the level density. We carried out this calculation in two steps; in the first step we evaluated the particle-number projection by a saddle-point approximation with respect to $\alpha_{p}$ and $\alpha_{n}$, and in a second step we evaluated the canonical to micro-canonical integration 


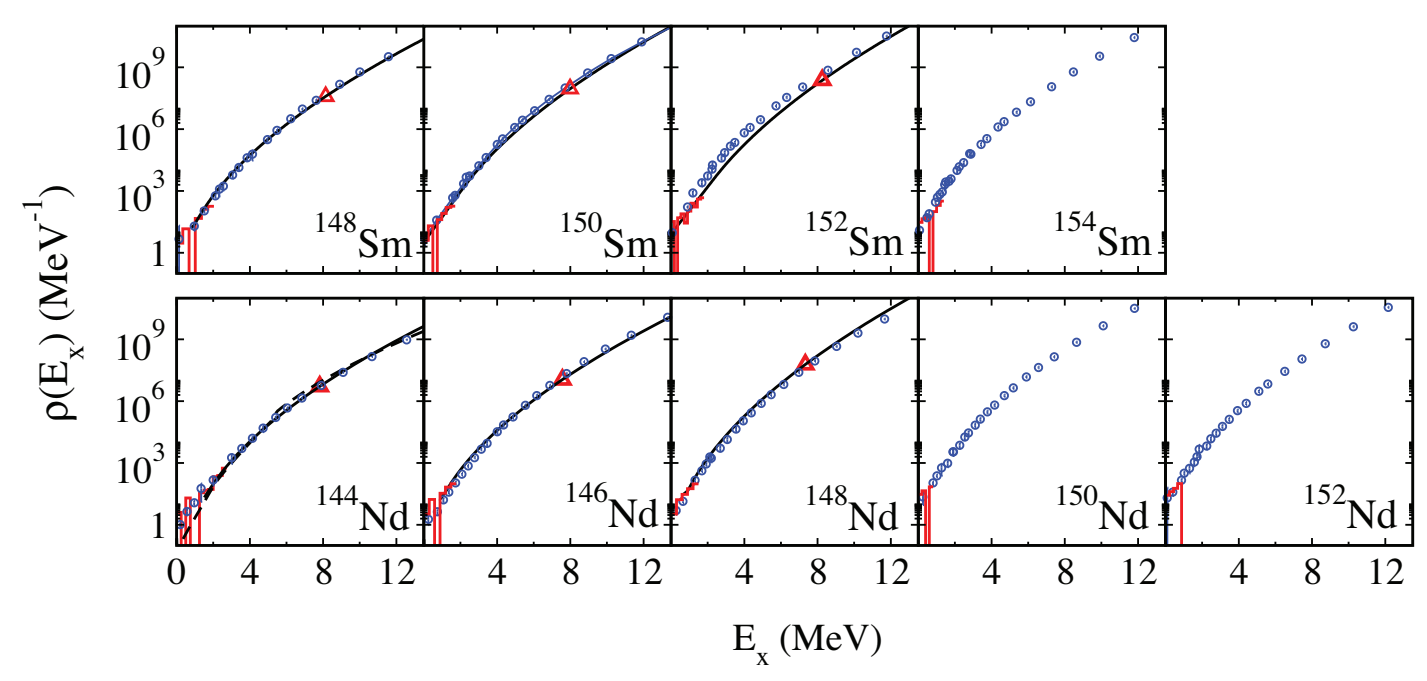

Figure 3. State densities in even-mass samarium ${ }^{148-154} \mathrm{Sm}$ and neodymium isotopes ${ }^{144-152} \mathrm{Nd}$. The SMMC results (solid circles) are compared with experimental data: level counting at low excitation energies (histograms) and neutron resonance data (triangles) [21]. The solid lines were computed using the empirical back-shifted Fermi gas formula with parameters determined from the experiments. Adapted from Refs. [10] and [20]

in (11) by a saddle point approximation with respect to $\beta$. The level density is then approximated by Eq. (12) in which the canonical entropy is

$$
S_{c}\left(\beta, N_{p}, N_{n}\right)=S_{g c}-\ln \zeta+\beta \delta E .
$$

with $S_{g c}$ being the grand canonical entropy. Here $\zeta$ is given by

$$
\zeta=2 \pi\left|\frac{\partial\left(N_{p}, N_{n}\right)}{\partial\left(\alpha_{p}, \alpha_{n}\right)}\right|^{1 / 2},
$$

and $\alpha_{p}, \alpha_{n}$ are determined by the 2-D saddle-point conditions $N_{i}=\partial \ln Z_{g c} / \partial \alpha_{i}(i=p, n)$. The correction $\beta \delta E$ with $\delta E=-d \ln \zeta / d \beta$ is absent in the usual 3-D saddle-point approximation.

\subsection{Entropies}

${ }^{162} \mathrm{Dy}$ is a deformed nucleus with a weak pairing condensate and the appropriate mean-field theory is the finite-temperature HF approximation. ${ }^{148} \mathrm{Sm}$ on the other hand is a spherical nucleus with strong pairing condensate, and the appropriate mean-field theory is the finite-temperature HFB approximation. In Figs. 4 and 5 we show the various entropy functions vs. $\beta$ for ${ }^{162}$ Dy and ${ }^{148} \mathrm{Sm}$, respectively.

The saddle-point approximation to particle-number projection breaks down when the particlenumber fluctuations are small. This happens in ${ }^{162}$ Dy using the HF approximation for $\beta$ above $\sim 5 \mathrm{MeV}^{-1}$. We then replace the saddle-point expression by a discrete Gaussian approximation discussed in Ref. [22]. In this approximation, $\zeta \rightarrow 1$ at large $\beta$ values, and the entropy (13) vanishes approximately in this limit. In SMMC, the entropy at low temperatures (that are still above $\sim 0.05 \mathrm{MeV}$ ) is finite and is well described by the contribution from the ground-state rotational band $S_{\text {rot }}=1+\ln \left(\mathcal{I}_{\mathrm{gs}} T / \hbar^{2}\right)$. 

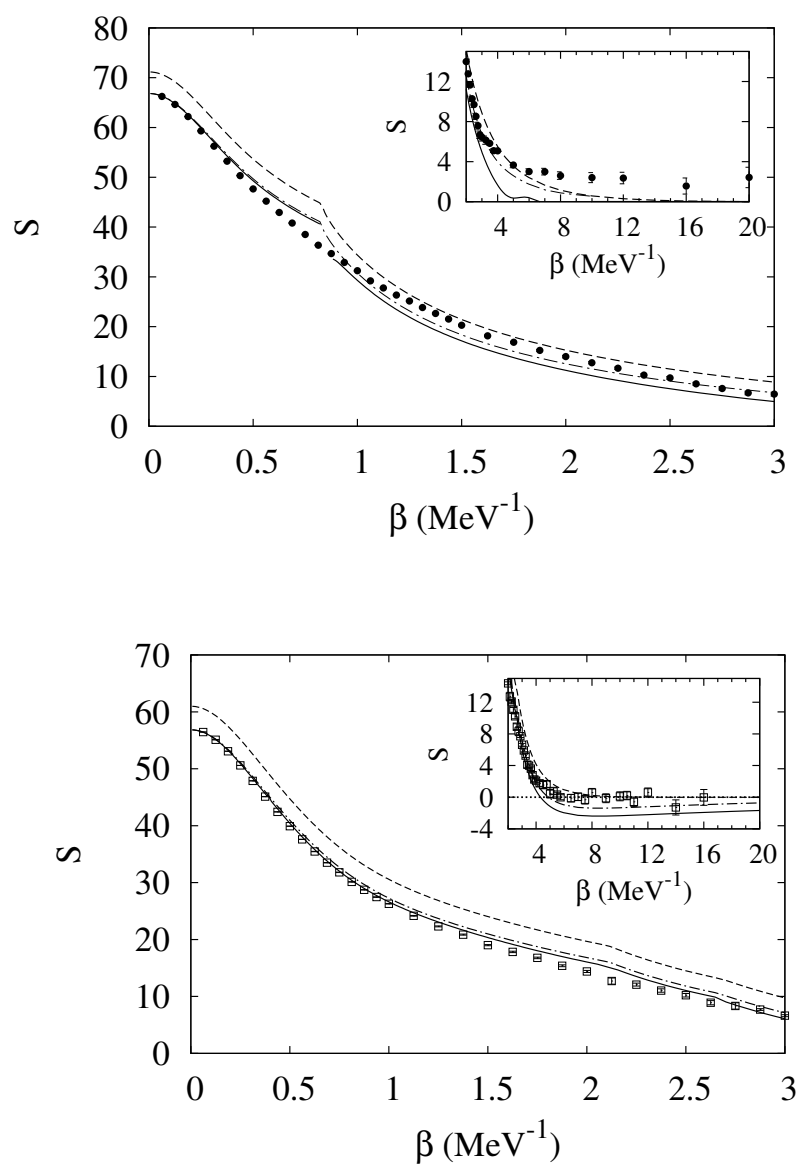

Figure 4. Entropy vs. inverse temperature $\beta$ in ${ }^{162}$ Dy. The SMMC entropy (solid circles) is compared with the grand canonical HF entropy (dashed line), the approximate canonical entropy defined in Eq. (13) (solid line), and the approximate canonical entropy obtained in the 3-D saddle-point approximation without the $\beta \delta E$ correction (dashed-dotted line). The calculations use the discrete Gaussian approximation [22]. The inset shows the large $\beta$ values. Adapted from Ref. [22].

Figure 5. Entropy vs. inverse temperature $\beta$ in ${ }^{148} \mathrm{Sm}$. The SMMC entropy (open squares) is compared with entropies obtained in the HFB approximation. The convention for the lines is as in Fig. 4 except that we use the finite-temperature HFB approximation. Taken from Ref. [22].

In ${ }^{148} \mathrm{Sm}$ we use the HFB approximation, in which the pairing condensate violates particle-number conservation and we find a negative approximate canonical entropy at low temperatures.

\subsection{Level densities}

Here we benchmark the finite-temperature mean-field level densities in comparison with the SMMC densities. The SMMC results are exact (up to statistical errors) for our model Hamiltonian except for the saddle-point approximation (12), which is also used in the finite-temperature mean-field approximations. In Fig. 6 we show the SMMC state density of ${ }^{162}$ Dy in comparison with the approximate canonical HF level density. We observe a large enhancement of the SMMC state density in comparison with the HF density that eventually disappears in the vicinity of the shape transition energy. The HF density measures the density of intrinsic states while SMMC includes also rotational bands that are built on top of the intrinsic band heads, hence the observed enhancement. A simple model that estimates the contribution of rotational bands [23], leads to overestimating the SMMC density around the neutron separation energy. Assuming a spin cutoff model [24] for the spin distribution with rigidbody moment of inertia gives a mean level spacing of $D=0.5 \mathrm{eV}$ for the $s$-wave resonances. The SMMC result of $D=2.4 \pm 0.3 \mathrm{eV}$ is consistent with the experimental result of $D=2.4 \mathrm{eV}$ [21]. 
The single-particle HF spectrum at the neutron separation energy is similar to the ground-state HF spectrum and a frozen potential model in which the single-particle spectrum is fixed (and taken from HF at $T=0$ ) gives a good approximation for the HF density at the neutron separation energy.

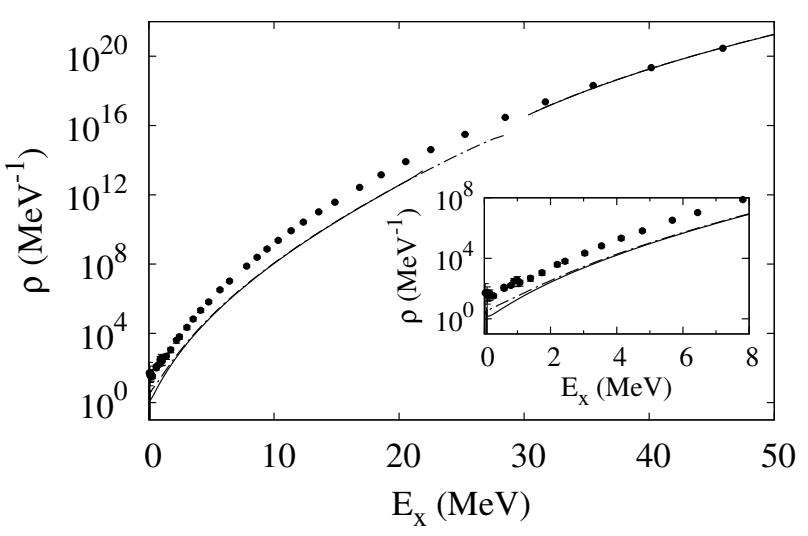

Figure 6. State densities vs. excitation energy $E_{x}$ in ${ }^{162}$ Dy. The SMMC state density (solid circles) is compared with the HF density calculated from (12) using the approximate canonical entropy defined in Eq. (13) (solid line), and the approximate level density obtained without the $\delta E$ correction in the approximate energy and canonical entropy (dashed-dotted line). The inset shows lower values of the excitation energy. Taken from Ref. [22].

In Fig. 7 we compare the SMMC state density of ${ }^{148} \mathrm{Sm}$ with the HFB results. For the neutron resonance spacing we find the HFB value of $D=4.1 \mathrm{eV}$ to be in good agreement with SMMC value of $D=3.7 \mathrm{eV}$ and the experimental value of $D=5.7 \mathrm{eV}$. The neutron separation energy of $\sim 8.2$ $\mathrm{MeV}$ is above the pairing transition where the HFB approximation reduces to the HF approximation and the HFB just resets the scale of the ground-state energy by the pairing correlation energy. This provides some justification to the back-shifted Fermi gas model.

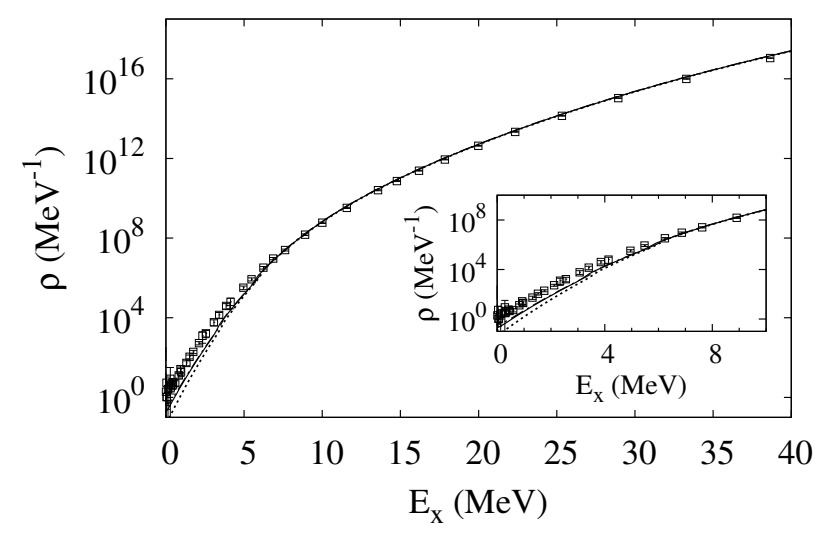

Figure 7. State densities vs. excitation energy $E_{x}$ in ${ }^{148} \mathrm{Sm}$. The SMMC state density (open squares) is compared with the HFB density calculated from (12) using the approximate canonical entropy defined in Eq. (13) (solid line), and the approximate level density obtained when $\zeta$ is calculated from particle-number fluctuations (dotted line) [22]. The inset shows lower values of the excitation energy. Taken from Ref. [22].

\section{Nuclear deformation in the $\mathrm{Cl}$ shell model}

The modeling of dynamical nuclear processes, such as fission, often requires knowledge of the level density as a function of deformation [25]. Nuclear deformation, however, is a concept introduced in the framework of a mean-field approximation that breaks rotational invariance of the nuclear Hamiltonian. The challenge is to study nuclear deformation and calculate statistical properties of nuclei 
as a function of deformation in an approach that preserves rotational invariance, such as the CI shell model.

Here we focus on the quadrupole deformation. In Sec. 6.1 we discuss the distribution of the axial quadrupole operator in the laboratory frame and show that it provides a model-independent signature of nuclear deformation [26]. In Sec. 6.2 we describe how we can calculate the distribution of the intrinsic quadrupole deformation within a framework that preserves rotational symmetry.

\subsection{Quadrupole distributions in the laboratory frame}

The axial quadrupole operator $\hat{Q}_{20}=\sum_{i}\left(2 z_{i}^{2}-x_{i}^{2}-y_{i}^{2}\right)$ does not commute with the Hamiltonian $\hat{H}$. Its distribution $P_{T}(q)$ in the laboratory frame at temperature $T$ is given by

$$
P_{T}(q)=\sum_{n} \delta\left(q-q_{n}\right) \sum_{m}\langle q, n \mid e, m\rangle^{2} e^{-\beta e_{m}},
$$

where $|q, n\rangle$ are eigenstates of $\hat{Q}_{20}$ satisfying $\hat{Q}_{20}|q, n\rangle=q_{n}|q, n\rangle$ and similarly for $|e, m\rangle$. The spectrum of $\hat{Q}_{20}$ in the truncated CI shell model is discrete.

\subsubsection{Axial quadrupole projection}

The distribution $P_{T}(q)$ can be calculated in SMMC using its Fourier representation

$$
P_{T}(q) \equiv \frac{\operatorname{Tr}\left[\delta\left(\hat{Q}_{20}-q\right) e^{-\beta \hat{H}}\right]}{\operatorname{Tr} e^{-\beta \hat{H}}}=\frac{\int_{-\infty}^{\infty} \frac{d \varphi}{2 \pi} e^{-i \varphi q} \operatorname{Tr}\left(e^{i \varphi \hat{Q}_{20}} e^{-\beta \hat{H}}\right)}{\operatorname{Tr} e^{-\beta \hat{H}}}
$$

together with the HS representation (1). In practice we use a discretized version of the Fourier transform, in which the interval $\left[-q_{\max }, q_{\max }\right]$ is divided into $2 M+1$ intervals of length $\Delta q=2 q_{\max } /(2 M+1)$. For a given configuration $\sigma$ of the auxiliary fields, we have

$$
\operatorname{Tr}\left[\delta\left(\hat{Q}_{20}-q\right) \hat{U}_{\sigma}\right] \approx \frac{1}{2 q_{\max }} \sum_{k=-M}^{M} e^{-i \varphi_{k} q_{m}} \operatorname{Tr}\left(e^{i \varphi_{k} \hat{Q}_{20}} \hat{U}_{\sigma}\right),
$$

where $q_{m}=m \Delta q(m=-M, \ldots, M)$ and $\varphi_{k}=\pi k / q_{\max }(k=-M, \ldots, M)$ are quadrature points.

Since $\hat{Q}_{20}$ is a one-body operator we can use the group property to represent the Fock space operator $e^{i \varphi_{k} \hat{Q}_{20}} \hat{U}_{\sigma}$ in the single-particle space by an $N_{s} \times N_{s}$ matrix $e^{i \varphi_{k} \mathbf{Q}_{20}} \mathbf{U}_{\sigma}$, where $\mathbf{Q}_{\mathbf{2 0}}$ is the axial quadrupole matrix in the single-particle space. In analogy with Eq. (3), the grand canonical traces in Eq. (17) are given by

$$
\operatorname{Tr}\left(e^{i \varphi_{k} \hat{Q}_{20}} \hat{U}_{\sigma}\right)=\operatorname{det}\left(1+e^{i \varphi_{k} \mathbf{Q}_{20}} \mathbf{U}_{\sigma}\right) .
$$

\subsubsection{Results}

In Fig. 8 we show the distributions $P_{T}(q)$ calculated with SMMC for ${ }^{154} \mathrm{Sm}$ at three temperatures. At a low temperature $(T=0.1 \mathrm{MeV})$ close to the ground state, the distribution is skewed and it becomes Gaussian like at high temperatures (see, e.g., at $T=4 \mathrm{MeV}$ ). At $T=0.1 \mathrm{MeV}$ the distribution is in good qualitative agreement with that of a prolate rigid rotor with an intrinsic quadrupole moment equal to the value found in the ground-state HFB solution (dashed line). The intermediate temperature of $T=1.2 \mathrm{MeV}$ is close to the shape transition temperature in HFB from a deformed to spherical shape. This distribution is still skewed, suggesting that deformation effects survive above the mean-field shape transition temperature.

In Fig. 9 we show such distributions $P_{T}(q)$ for ${ }^{148} \mathrm{Sm}$. In HFB, this nucleus is spherical in its ground-state solution, and we observe a Gaussian-like distribution already at the low temperature of $T=0.1 \mathrm{MeV}$. We conclude that $P_{T}(q)$ is a model-independent signature of deformation. 


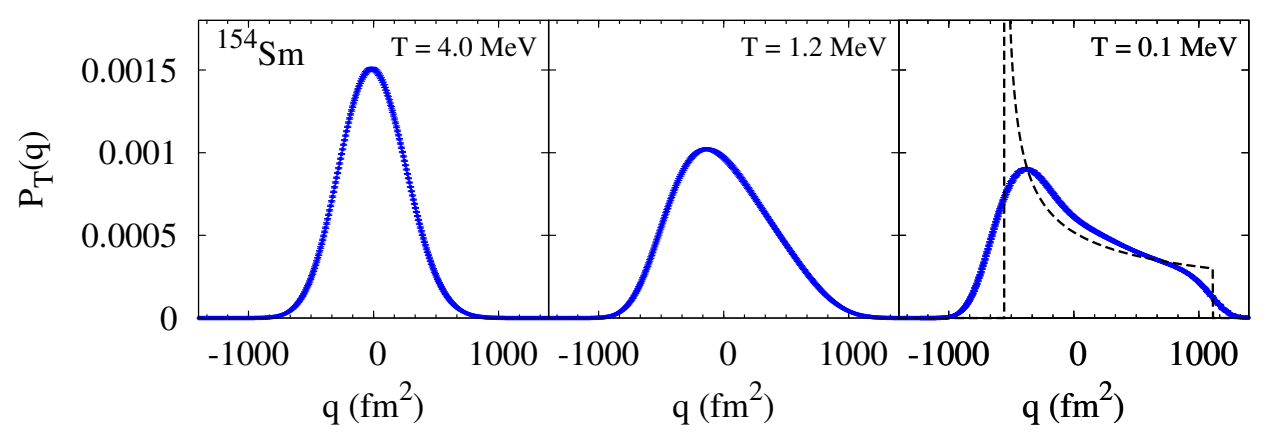

Figure 8. Axial quadrupole distributions $P_{T}(q)$ in the laboratory frame for ${ }^{154} \mathrm{Sm}$ at three temperatures.

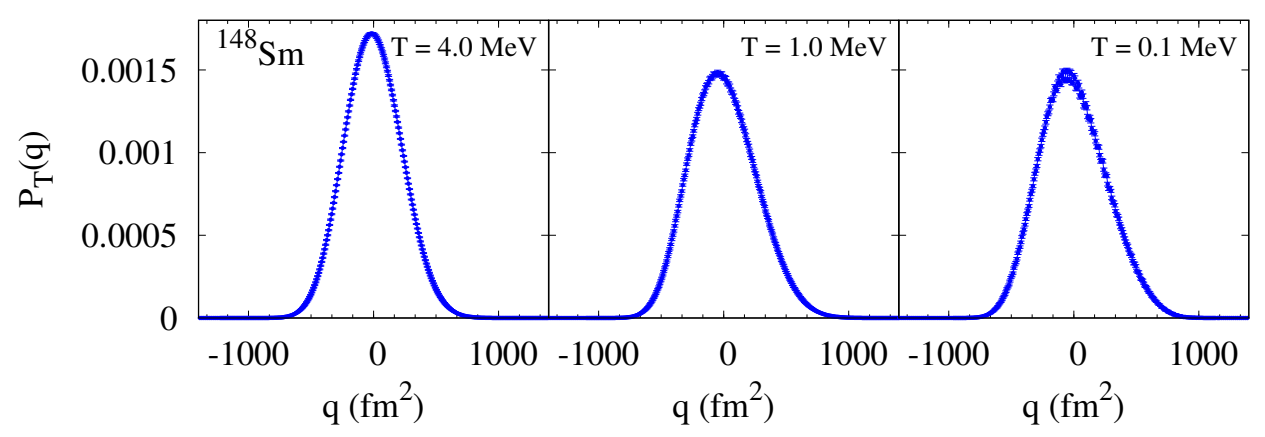

Figure 9. Axial quadrupole distributions $P_{T}(q)$ in the laboratory frame for ${ }^{148} \mathrm{Sm}$ at three temperatures.

\subsection{Quadrupole distributions in the intrinsic frame}

The quadrupole deformation is described by a second-rank tensor with spherical components $q_{2 \mu}$ $(\mu=-2, \ldots, \mu)$. In the intrinsic frame, characterized by a set of Euler angles $\Omega$, the deformation parameters are denoted by $\beta, \gamma$. Information on $\beta, \gamma$ can be obtained from the expectation values of rotationally invariant combinations of the quadrupole tensor, known as quadrupole invariants [27]. The distribution $P_{T}$ is invariant under rotations and therefore in the intrinsic frame it depends only on $\beta, \gamma$. We expand $-\ln P_{T}(\beta, \gamma)$ in the quadrupole invariants. These invariant are unique up to fourth order and are given by $\beta^{2}, \beta^{3} \cos (3 \gamma)$ and $\beta^{4}$. To this order we have

$$
-\ln P_{T}(\beta, \gamma)=N+A \beta^{2}-B \beta^{3} \cos 3 \gamma+C \beta^{4}+\ldots,
$$

where $A, B, C$ are temperature-dependent parameters and $N$ is a normalization constant. We can determine $A, B, C$ from the expectation values of the above three invariants, which in turn can be calculated from the moments of $\hat{Q}_{20}$ in the laboratory frame [26]

$$
\langle\hat{Q} \cdot \hat{Q}\rangle=5\left\langle\hat{Q}_{20}^{2}\right\rangle ;\langle(\hat{Q} \times \hat{Q}) \cdot \hat{Q}\rangle=-5 \sqrt{\frac{7}{2}}\left\langle\hat{Q}_{20}^{3}\right\rangle ;\left\langle(\hat{Q} \cdot \hat{Q})^{2}\right\rangle=\frac{35}{3}\left\langle\hat{Q}_{20}^{4}\right\rangle
$$

The moments of $\hat{Q}_{20}$ in Eq. (20) can be directly calculated from the SMMC distributions $P_{T}(q)$. The expressions for the expectation values of the invariants in terms of $P_{T}(\beta, \gamma)$ in Eq. (19) requires the 
metric $\prod_{\mu} d q_{2 \mu}$, which in the intrinsic frame parameters is given by

$$
\prod_{\mu} d q_{2 \mu}=\frac{1}{2} \beta^{4}|\sin 3 \gamma| d \beta d \gamma d \Omega
$$

with $\beta \geq 0$ and $0 \leq \gamma \leq \pi / 3$. To test the accuracy of the expansion (19), we expressed the invariants in terms of $q_{2 \mu}$ in the laboratory frame and integrated over $q_{2 \mu}$ for all $\mu \neq 0$ to find the marginal distribution $P_{T}\left(q_{20}\right)$. We found this marginal distribution to be essentially indistinguishable from the SMMC distribution $P_{T}(q)$.

In Fig. 10 we show $-\ln P_{T}(\beta, \gamma=0)$ as a function of $\beta$ for ${ }^{154} \mathrm{Sm}$ at three temperatures. We observe that these curves mimic the deformed to spherical shape transition in the Landau mean-field theory of shape transitions $[28,29]$, in which the quadrupole tensor $q_{2 \mu}$ is the order parameter. However, the curves here are calculated within the CI shell model approach that preserves rotational symmetry.
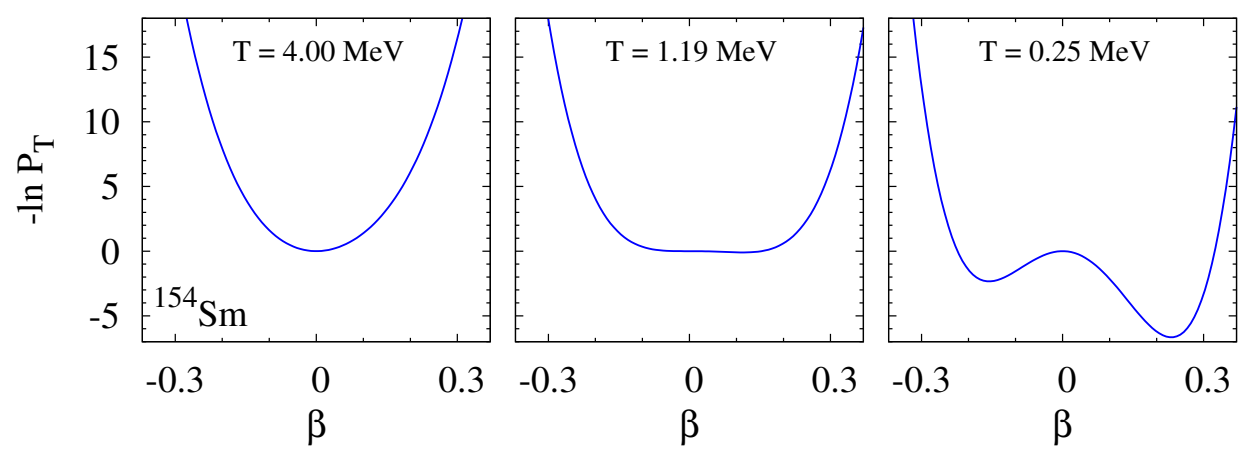

Figure 10. $-\ln P_{T}(\beta, \gamma=0)$ in Eq. (19) as a function of axial deformation $\beta$ for ${ }^{154} \mathrm{Sm}$ at three temperatures. The parameters $A, B, C$ are determined from the expectation values of the three lowest-order quadrupole invariants.

The quadrupole distributions $P_{T}(\beta, \gamma)$ can in principle be converted to level densities $\rho\left(E_{x}, \beta, \gamma\right)$ as a function of intrinsic deformation by using the saddle-point approximation.

\section{Conclusion}

The SMMC method is a powerful method to calculate statistical and collective properties of nuclei in very large model spaces in the presence of correlations. We discussed recent applications of the method in heavy nuclei for identifying various types of collectivity and for calculating state densities. We also studied the SMMC distributions of the axial quadrupole operator in the laboratory frame and showed that they provide model-independent signatures of deformation. Finally we outlined a method to determine the quadrupole distributions in the intrinsic frame using the rotationally invariant framework of the CI shell model. This method can be used to determine level densities as a function of intrinsic deformation.

\section{Acknowledgements}

This work was supported in part by the DOE grant Nos. DE-FG-0291-ER-40608 and DE-FG0200ER411132, by Grant-in-Aid for Scientific Research (C) No. 25400245 by the JSPS, Japan, and 
by the Turkish Science and Research Council (TÜBITAK) grant No. ARDEB-1001-110R004 and ARDEB-1001-112T973. The research presented here used resources of the National Energy Research Scientific Computing Center, which is supported by the Office of Science of the U.S. Department of Energy under Contract No. DE-AC02-05CH11231. It also used resources provided by the facilities of the Yale University Faculty of Arts and Sciences High Performance Computing Center.

\section{References}

[1] W. Hauser and H. Feshbach, Phys. Rev. 87, 366 (1952).

[2] S. Hilaire and S. Goriely, Nucl. Phys. A 779, 63 (2006).

[3] G.H. Lang, C.W. Johnson, S.E. Koonin, and W.E. Ormand, Phys. Rev. C 48, 1518 (1993).

[4] Y. Alhassid, D.J. Dean, S.E. Koonin, G.H. Lang, and W.E. Ormand, Phys. Rev. Lett. 72, (1994) 613.

[5] S.E. Koonin, D.J. Dean, and K. Langanke, Phys. Rep. 278, 2 (1997).

[6] Y. Alhassid, Int. J. Mod. Phys. B 15, 1447 (2001).

[7] J. Hubbard, Phys. Rev. Lett. 3, 77 (1959); R.L. Stratonovich, Dokl. Akad. Nauk. S.S.S.R. 115, 1097 (1957).

[8] W.E. Ormand, D.J. Dean, C.W. Johnson, G.H. Lang, and S.E. Koonin, Phys. Rev. C 49, 1422 (1994).

[9] Y. Alhassid, L. Fang and H. Nakada, Phys. Rev. Lett. 101, 082501 (2008).

[10] C. Özen, Y. Alhassid and H. Nakada, Phys. Rev. Lett. 110, 042502 (2013).

[11] M. Dufour and A.P. Zuker, Phys. Rev. C 54, 1641 (1996).

[12] C. Özen, Y. Alhassid and H. Nakada, Phys. Rev. C 91 (2015) 034329.

[13] Y. Alhassid, M. Bonett-Matiz, A. Mukherjee, H. Nakada, and C. Özen, Journal of Physics: Conference Series 580, 012009 (2015).

[14] H. Nakada and Y. Alhassid, Phys. Rev. Lett. 79, 2939 (1997).

[15] Y. Alhassid, S. Liu and H. Nakada, Phys. Rev. Lett. 83, 4265 (1999).

[16] Y. Alhassid, G. F. Bertsch, S. Liu, and H. Nakada, Phys. Rev. Lett. 84, 4313 (2000).

[17] Y. Alhassid, G.F. Bertsch, and L. Fang, Phys. Rev. C 68, 044322 (2003).

[18] Y. Alhassid, S. Liu and H. Nakada, Phys. Rev. Lett. 99, 162504 (2007).

[19] A. Bohr and B. R. Mottelson, Nuclear Structure, Vol. 1 (Benjamin, New York, 1969).

[20] Y. Alhassid Y, C. Özen, and H. Nakada, Nuclear Data Sheets 118, 233 (2014).

[21] R. Capote, M. Herman, P. Obložinský, P.G. Young, S. Goriely, T. Belgya, A.V. Ignatyuk, A.J. Koning, S. Hilaire, V.A. Plujko, M. Avrigeanu, O. Bersillon, M.B. Chadwick, T. Fukahori, Zhigang Ge, Yinlu Han, S. Kailas, J. Kopecky, V.M. Maslov, G. Reffo, M. Sin, E.Sh. Soukhovitskii, and P. Talou, Nuclear Data Sheets, 110, 3107 (2009).

[22] Y. Alhassid, G.F. Bertsch, C.N. Gilbreth, and H. Nakada, arXiv:1512.03773.

[23] S. Bjørnholm, A. Bohr and B.R. Mottelson, Physics and Chemistry of Fission 1973 (IAEA, Austria, 1974), p. 367.

[24] T. Ericson, Adv. Phys. 9, 425 (1960).

[25] G.F. Bertsch and J.M. Mehlhaff, arXiv:1511.01936.

[26] Y. Alhassid, C.N. Gilbreth, and G.F. Bertsch, Phys. Rev. Lett. 113, 262503 (2014).

[27] K. Kumar, Phys. Rev. Lett. 28, 249 (1972); D. Cline, Ann. Rev. Nucl. Part. Sci. 36, 683 (1986).

[28] S. Levit and Y. Alhassid, Nucl. Phys. A 413, 439 (1984).

[29] Y. Alhassid, S. Levit and J. Zingman, Phys. Rev. Lett. 57, 539 (1986). 